\title{
The point-of-care testing in the emergency department
}

\author{
Paolo Carraro \\ Department of Laboratory Medicine, ULSS3 Serenissima, Venice, Italy
}

\begin{abstract}
The decentralization of analysis at the emergency room is a well-established practice, in particular for the use of blood gas analysis. Recently, many other analyzers have been proposed, with rapid methods that can potentially reduce the response time of the tests. Here we consider the various analyzers that can be used at the bedside, their advantages and limits, the related scientific evidences. Finally, we discuss their impact both on patient care and on accelerating the patient's flow in the emergency room.
\end{abstract}

\section{Introduction}

Quick availability of laboratory tests is a critical issue for the Emergency Department (ED). Continuous request for a shorter turnaround time (TAT) is frequently experienced by almost all laboratories that perform urgent exams. Two are the reasons for this need: first, the possibility of an early intervention in critically ill patients. Second, the purpose of facilitating the taking charge, even in non-critical subjects. Therefore the adoption of the point-of-care testing (POCT) can also contribute to containment of the crowding of waiting rooms. In the case of the most serious patients the choice of a blood gas analyzer placed in the examination room in the main hospitals has made a major contribution to the treatment of dyspnea, metabolic disorders, sepsis, and renal failure. So, the

Correspondence: Paolo Carraro, Department of Laboratory Medicine, ULSS3 Serenissima, 30122 Venice, Italy.

Tel.: +39.041.5294244 - Fax: +39.041.5294717.

E-mail: paolo.carraro@aulss3.veneto.it

Key words: Point-of-care testing; Turnaround time; Near patient testing; Evidence-based medicine; Laboratory medicine.

Conflict of interest: the author declares no potential conflict of interest.

Funding: none.

Conference presentation: some of these data were presented at the National Congress of Academy of Emergency Medicine and Care, Pisa, Italy, November $9^{\text {th }} 2018$.

Received for publication: 2 January 2019.

Revision received: 15 February 2019.

Accepted for publication: 19 February 2019.

This work is licensed under a Creative Commons Attribution 4.0 License (by-nc 4.0).

(C) Copyright: the Author(s), 2019

Licensee PAGEPress, Italy

Emergency Care Journal 2019; 15:8019

doi:10.4081/ecj.2019.8019
POCT in emergency is an experience consolidated for many years. Over the last decades a lot of new, easy to use, speedy devices for several analytes have been proposed on the market. There are numerous methods available from different suppliers for various laboratory analyses: blood gas, electrolytes, basic biochemistry, hematology, coagulation, inflammation markers, cardiac markers, toxicology, urinalysis, pregnancy, infections and serology (Table 1). Development in this field is constantly expanding and the global point-of-care diagnostics market is projected to reach 38.13 billion USD by 2022 from 23.71 billion USD in $2017 .{ }^{1}$

To test at the bedside is not a new as laboratory medicine was born precisely in this way, with the examination of urine and other biological fluids directly near the patient; then in the ' 30 s testing was moved to suitable premises with dedicated instruments. Later the laboratories grew and became a discipline with a complex structure but the reverse process of returning to the clinical department is a more recent event that requires standardized procedures and quality assurance.

It is usually performed by staff without laboratory training, although also encompasses patient self-monitoring. POCT provides rapid results near the patient which can be acted upon immediately. By contrast, analysis in the clinical laboratory could at times incur significant delays in TAT.

The estimation of TAT is often referred to the intra-laboratory phases which are more easily extracted from the informatics databases of the laboratories. It would instead be desirable to always refer to the diagnostic cycle described by Lundberg that includes the complete pathway from the clinical question up until the clinical action, both diagnostic or therapeutic ${ }^{2}$ (Figure 1). If we consider this overall time the clinical laboratories can keep the TAT under 60 minutes with difficulty. In fact, the time required for blood collection, identification of the tubes, transport to the laboratory through the pneumatic tube system takes about 5-10 minutes. In the laboratory the phases depend on the workload and the specific tests required: an average values of at least 35 minutes are usual. From this description it is clear how the 90th percentile easily settles on 70-100 minutes. It is therefore understandable that the POCT test has in any case an advantage of unquestionable speed, at least as long as one test is requested at a time.

\section{Is the use of point-of-care testing analyzers in emergency department supported by efficacy evi- dences?}

The clinical scenarios where the POCT were investigated by evidence-based studies is limited to some specific cases such as: remote rural communities, primary care medicine, self-monitoring, pharmacies, drugs of abuse and finally the ED. ${ }^{3}$ In this field some papers explored the specific diagnostic conditions related to dyspnea (Brain Natriuretic Peptide), thromboembolism (D-dimer), sepsis (lactate), metabolism (blood gas and electrolytes), pregnancy (human Chorionic Gonadotropin) and acute coronary syndrome with the use of troponins. Most of the studies do not reach high qual- 
ity levels and express opinions of experts based on limited series. ${ }^{3} \mathrm{~A}$ study using a randomized and prospective methodology in 1728 subjects, verified the impact of a basic biochemical profile in a British emergency room; a half of patients were randomly managed with a rapid test, while others performed the tests at a centralized laboratory. ${ }^{4}$ Unfortunately, a study with these characteristics has not been conducted since 1998, but we can still consider it valid in the main conclusions: TAT has been shown to be reduced; in about $7 \%$ of patients appropriate therapy was started earlier, but the length of stay (LOS) in ED has not improved and overall mortality has not decreased. Therefore, some organizational advantage was demonstrated with no improvement of clinical outcome.

A more recent experience was evidenced by Levandrowski at the Massachusetts General Hospital: typical POCT instruments were used near an ED, but managed directly by laboratory staff (glucose, coagulation, urine pregnancy, dipstick urinalysis, creatine kinase-MB and Cardiac Troponin (cTn). ${ }^{5}$ Some of the tests decreased ED LOS (urine testing, rapid D-dimer), whereas other test results allowed rapid diagnosis and triage of patients presenting to the ED. We can conclude that rapid analytical methodologies can offer some clinical advantages, even if the evidence is not fully demonstrated. Figure 2 shows that the reduction in laboratory TAT contributes only partially to a potential reduction of ED LOS because the variables that determine it are several others.

\section{Laboratory exams in disadvantaged areas}

The use of POCT is particularly suitable where the first aid takes place in remote, rural areas, smaller islands, aircraft, ships, environments with low atmospheric pressure or gravitation, in countries without a land rescue system. In all these conditions, efficacy studies and evaluations were carried out using portable analytical systems. An experience in New Zealand, for example, has shown in particular an advantage of POCT in the treatment of less severe patients, where an immediate test made it possible to reduce transfers to the base hospital by $62 \%$ with an increase in discharges. ${ }^{6}$ Other positive clinical results have been reported with moderate levels of evidence in the management of acute coronary syndromes (ACS) in rural areas in Australia through the use of troponin determination in the field. ${ }^{7}$

\section{Point-of-care testing troponins and acute coronary syndrome}

Chest pain is one of the most relevant presenting symptoms in $\mathrm{ED}$ and the consequent determination of $\mathrm{cTn}$ is essential to the ACS classification when the ECG pattern is not clear. Clinical laboratories have continuously improved the performance of this test, in particular the analytical sensitivity and consequently its diagnostic precocity. High-sensitivity cTn assays (hs-cTn), as defined today, should have coefficient of variation $\leq 10 \%$ at the 99 th percentile of cTn concentrations seen in healthy individuals. In addition, the measured concentration should exceed the limit of detection in at least $50 \%$ (ideally $95 \%$ ) of healthy subjects. ${ }^{8}$ POCT methods are a good alternative to reducing TAT, but their analytical

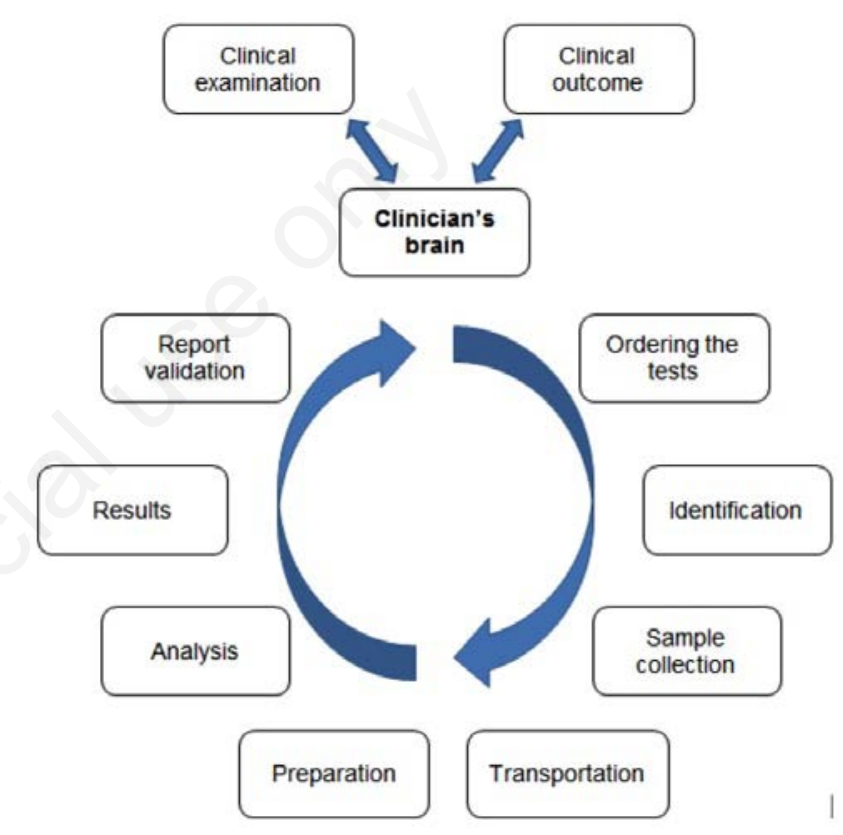

Figure 1. Steps in the brain-to-brain loop defining the therapeutic turnaround time.

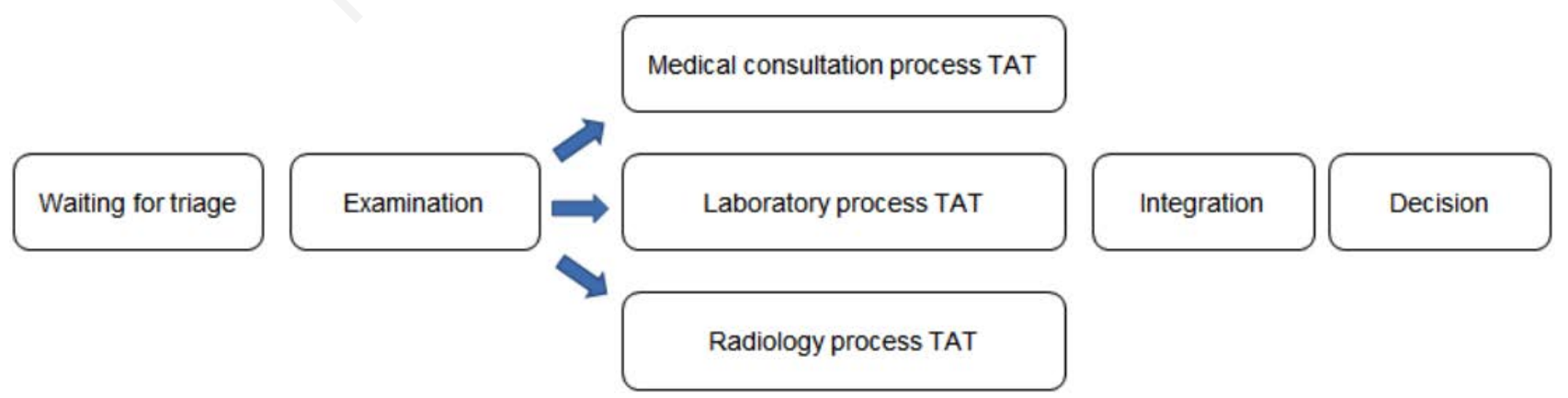

ED-LOS

Figure 2. Length of stay in emergency department: some of the main variables. TAT, therapeutic turnaround time; ED-LOS, emergency department- length of stay. 
sensitivity is not improved at the same speed as laboratory systems. ${ }^{9}$ Only recently there is a POCT test on the market that the manufacturer affirms in accordance to the definition of high sensitivity, the first cTn high sensitivity cleared by the Food and Drug Administration, as confirmed in a preliminary study. ${ }^{10}$ Other methods are under development, but right now the choice of an instrument that complies with the recommendations is very limited at this time. ${ }^{9}$ Most published studies have compared the POCT cTn with conventional cTn assays performed at the laboratory. Little data are available on the comparison of POCT cTn with a conventional hs-cTn. Some manufacturers of cardiac POCT instruments propose the association of other markers in various combinations, such as Creatine Kinase MB, Myoglobin, Brain Natriuretic Peptide, D-dimer, C-reactive protein. Some of these are no longer recommended by cardiology guidelines and can often be considered as completely inappropriate. Only the combination of a hscTn with Copeptin seems to have a greater diagnostic accuracy, but this does not happen with cTn of lower analytical quality. ${ }^{11}$

\section{Point-of-care testing in other clinical conditions}

The combination of D-dimer test with clinical assessment is effective to exclude venous thrombo-embolism in low pre-test probability patients. This statement is particularly valid if the test has negative predictive value of more than $98 \%$ and a TAT of less than 38 minutes. ${ }^{12}$ Some assays dedicated to the POCT have these characteristics, although some papers have discussed the value of tests with analytical performance lower than those of the laboratory. Unfortunately, the conditions present in an ED often prompt doctors to request this test inappropriately. ${ }^{13}$

In the case of suspected sepsis, a rapid TAT for both tests and treatments is recommended by the Surviving Sepsis Campaign guidelines specifically indicating a limited number of laboratory tests such as Blood gas, Platelet count, Creatinine, Bilirubin and Lactic acid. ${ }^{14}$ These tests are related to the initial diagnosis of sepsis or septic shock by the Sequential Organ Failure Assessment score. There is no published evidence on the use of POCT for this specific diagnosis that cannot be resolved by using a single instrument. Moreover, we have to consider that several other laboratory markers are suitable to identify the type of patients who are often complicated by concurrent diseases: Leukocytes count, immature Granulocytes, coagulation tests, C-reactive protein, Procalcitonin, Presepsin, Urinalysis, microbiological assays and other fluid examinations. It is not possible to perform all these tests with a POCT methodology.

In another field, several rapid tests are used in ED to search for the drugs of abuse in urine. This represents a real analytical challenge as there are several categories of substances, sometimes with different metabolites and highly variable concentrations due to the different pharmacological potency. These simplified tests therefore have many limitations, present false positives and even more false negatives results. Furthermore, due to possible legal implications, they require subsequent confirmatory tests with definitive methods that are not suitable for clinical urgency. Because of these limited diagnostic performances, they should only be used in the case of elevated clinical suspicion of intoxication and not as a first screening test. There are no substantial data on the real clinical advantage offered using these tests in ED. ${ }^{15}$

In respiratory disorders the value of blood gas analysis in ED is established and indisputable, as well as the value of electrolytes in case of fever or dyspnea for other causes. In newborn or very young patients, some studies have evaluated the use of C-reactive protein: it is a marker whose limitations are known, but in these cases a considerable advantage is offered by the use of capillary blood which is obtained more easily and does not contribute to the development of iatrogenic anemia. More recently, rapid molecular tests have been proposed for the diagnosis of influenza and other viruses. Now at least one of these methods seems to be reliable but there is no evidence of efficacy in reducing the use of antibiotics. ${ }^{16}$

\section{Quality in point-of-care testing process}

One of the main aspects concerning the adoption of POCT systems is quality assurance. There is in fact a native quality, that is the one guaranteed by the manufacturer, with a method that is valid from an analytical point of view and with an instrument that prevents errors in use. Table 1 shows how, on average, the analytical performance is not always comparable with that provided by the laboratories evidencing its insufficiency. It is also important that correct procedures for internal quality control are adopted with suitable materials and an external quality audit, while the pre-ana-

Table 1. Mean analytical performance of common methods for point-of-care testing.

\begin{tabular}{ll}
\hline Analytes & Analytical performance \\
Blood gas and electrolytes & Optimal \\
Cardiac markers & Variable \\
\hline Basic biochemistry & Acceptable \\
Inflammation markers & Acceptable \\
\hline Complete blood count & Acceptable \\
Coagulation & Acceptable \\
\hline Pregnancy test & Acceptable \\
Urinalysis & Acceptable \\
\hline Infection, molecular & Acceptable \\
Serology & Acceptable \\
\hline Drugs of abuse & Limited \\
Toxicology & Limited \\
\hline
\end{tabular}

\section{Table 2. Typical errors in the point-of-care testing.}

\begin{tabular}{ll} 
Withdrawal from the wrong patient & Pre-analytical \\
Incorrect test tube or container & Pre-analytical \\
\hline Unwashed fingertip & Pre-analytical \\
Temporary patient instability & Pre-analytical \\
\hline Use of the second or third drop of capillary blood & Pre-analytical \\
Presence of air bubbles in syringe & Pre-analytical \\
\hline Choice of incorrect units of measurement & Analytical \\
Unsuitable collection site & Analytical \\
\hline Interference not recognized & Analytical \\
Procedural error & Analytical \\
\hline Quality control not performed & Analytical \\
Failure of quality control not recognized & Analytical \\
\hline Equipment maintenance not performed & Analytical \\
Misinterpretation of results & Post-analytical \\
\hline Delay on result communication & Post-analytical \\
Transcription error & Post-analytical \\
\hline
\end{tabular}


lytical and post-analytical phases require integrating the POCT into the quality system of the whole ED. For this purpose, the specific International Organization for Standardization (ISO) 22870:2017 document for this activity must be followed for each clarification. ${ }^{17}$ Rules and recommendations are provided for the training of personnel, their qualification to perform tests, maintenance procedures, technical validation of data and management of any panic values. The laboratory providing technical support must collaborate in all of this, interfacing the analyzers with the Laboratory Information System and ensuring the traceability of data.

\section{Errors and point-of-care testing}

Laboratory errors occur mainly in the pre-analytical and postanalytic phases, as numerous publications have shown. ${ }^{18,19}$ This is expected from the numerous safety processes implemented in laboratories through analytical quality control systems. Contrarily in POCT, analytical errors prevail $;{ }^{20}$ in fact, the pre-analytical phase is simplified, in particular when a primary container is not required with the biological fluid going directly to the analyzer. Conversely, the analytical phase is marked by two critical issues: the analytical method is limited because simplified to favor timeliness and the procedure is followed by nurses who have many other tasks. Table 2 shows the main types of errors that have been described in the POCT, divided into the different phases of the process. In the specific situation of the ED where there is a considerable crowd of users and frequent distractions to follow more urgent cases, the correct identification of the subject and the subsequent transcription of the results, however, remain at high risk of error. ${ }^{21}$ Two are the interventions needed for avoiding or limiting the high risks related to an activity that often induce an immediate clinical decision: first, implementation of a quality system (as the ISO 22870:2017) for the whole process, with particular attention to the staff competency; second, activation of a reliable informatics connection between the patient identification, analyzer, Laboratory Information System, reports production and consultation.

\section{Cost analysis}

Financial impact with the use of POCT also needs to be evaluated to consider both the costs to the system and the cost to the patient. POCT per se was not found to reduce costs, despite reducing LOS and admissions. In fact, we can attribute the rising costs not only to the consumables, but also to the work time of the nurse, the training, the quality control, the computer interfacing and the commitment required by the laboratory staff. Among the ceasing costs we must include some consumables for blood samples, transport of tubes, time dedicated to the laboratory work, maintenance and the cost of reagents and analyzers. The evaluation of benefits is more difficult, as are the partial and uncertain conclusions of literature. For all these considerations it is not possible to express any judgment now. ${ }^{22}$

\section{Conclusions}

Clinical studies with a high value of evidence have investigated only some applications of POCT exams in ED, so we have no clear proof of the comparison between the tests performed at the
Laboratory compared to decentralized tests.

POCT does not replace the clinical laboratory, but we must consider it as an extension of the laboratory that approaches the clinic and helps the ED staff in obtaining useful and safe reports in patient care. All this requires sharing and precise rules.

The need to accelerate the diagnostic process in ED is however very strong and requires continue experimenting with new technical solutions, albeit with the prudence required by the fragility of patients.

\section{References}

1. MarketsandMarkets ${ }^{\mathrm{TM}}$. Market research report. MD 2702. February 2018. Available from: https://www.marketsandmarkets.com/pdfdownloadNew.asp?id=106829185 Accessed: December 10, 2018.

2. Lundberg GD. Acting on significant laboratory results. JAMA 1981;245:1762-3.

3. Florkowski C, Don-Wauchope A, Gimenez N, et al. Point-ofcare testing (POCT) and evidence-based laboratory medicine (EBLM) - does it leverage any advantage in clinical decision making? Crit Rev Clin Lab Sci 2017;54:471-94.

4. Kendall J, Reeves B, Clancy M. Point of care testing: randomised controlled trial of clinical outcome. Br Med J 1998;316:1052-7.

5. Lewandrowski KFJ, Tochka L, Lee-Lewandrowski E. Implementation of a point-of-care satellite laboratory (kiosk) in the emergency department of an academic medical center: an 8-year experience at the Massachusetts General Hospital. Point of Care 2011;10:93-7.

6. Blattner K, Nixon G, Dovey S, et al. Changes in clinical practice and patient disposition following the introduction of pointof-care testing in a rural hospital. Health Policy 2010;96:7-12.

7. Tideman PA, Tirimacco R, Senior DP, et al. Impact of a regionalised clinical cardiac support network on mortality among rural patients with myocardial infarction. Med J Aust 2014;200:157-60.

8. Apple FS, Sandoval Y, Jaffe AS, Ordonez-Lianos J; IFCC Task Force on Clinical Applications of Cardiac Bio-Markers. Cardiac troponin assays: guide to understanding analytical characteristics and their impact on clinical care. Clin Chem 2017;63:73-81.

9. Suh D, Keller DI, Hof D, et al. Rule-out of non-ST elevation myocardial infarction by five point of care cardiac troponin assays according to the $0 \mathrm{~h} / 3 \mathrm{~h}$ algorithm of the European Society of Cardiology. Clin Chem Lab Med 2018;56:649-57.

10. Christenson RH, Mullins K, Duh SH. Validation of high-sensitivity performance for a United States Food and Drug Administration cleared cardiac troponin I assay. Clin Biochem 2018:56:4-10.

11. Lipinski MJ, Escarcega RO, D'Ascenzo F, et al. A systematic review and collaborative meta-analysis to determine the incremental value of copeptin for rapid rule-out of acute myocardial infarction. Am J Cardiol 2014;113:1581-91.

12. Marquardt U, Apau D. Point-of-care D-dimer testing in emergency departments. Emerg Nurse 2015;23:29-35.

13. Kristoffersen AH, Ajzner E, Bauça JM, et al. Pre- and post-test probabilities of venous thromboembolism and diagnostic accuracy of D-dimer, estimated by European clinicians working in emergency departments. Thromb Res 2017;159:19-23.

14. Rhodes A, Evans LE, Alhazzani W, et al. Surviving sepsis 
campaign: international guidelines for management of sepsis and septic shock: 2016. Intensive Care Med 2017;43:304-77.

15. Tenenbein M. Do you really need that emergency drug screen? Clin Toxicol 2009;47:286-91.

16. Gibson J, Schechter-Perkins EM, Mitchell P, et al. Multi-center evaluation of the $\operatorname{cobas}{ }^{\circledR}$ Liat ${ }^{\circledR}$ Influenza A/B \& RSV assay for rapid point of care diagnosis. J Clin Virol 2017;95:5-9.

17. ISO (International Organization for Standardization). Point-ofcare testing (POCT) - Requirements for quality and competence. Norm ISO 22870:2016. Geneva: International Organization for Standardization Publications; 2016. Available from: https://www.iso.org/standard/71119.html Accessed: December 10, 2018.

18. Carraro P, Zago T, Plebani M. Exploring the initial steps of the testing process: frequency and nature of pre-preanalytic errors. Clin Chem 2012;58:638-42.

19. Carraro P, Plebani M. Errors in a stat laboratory: types and frequencies 10 years later. Clin Chem 2007;53:1338-42.

20. Kazmierczak SC. Point-of-care testing quality: some positives but also some negatives. Clin Chem 2011;57:1219-20.

21. Carraro P, Plebani M. Post-analytical errors with portable glucose meters in the hospital setting. Clin Chim Acta 2009;404:65-7.

22. Fitzgerald P, Goodacre SW, Cross E, et al. Cost-effectiveness of point-of-care biomarker assessment for suspected myocardial infarction: the randomized assessment of treatment using panel assay of cardiac markers (RATPAC) trial. Acad Emerg Med 2011;18:488-95. 